\title{
Bacillus subtilis 168 Mutants Resistant to Arginine Hydroxamate in the Presence of Ornithine or Citrulline
}

\author{
By SIMON BAUMBERG* AND ANDREW MOUNTAIN \\ Department of Genetics, University of Leeds, Leeds LS2 9JT, UK
}

(Received 10 October 1983; revised 17 January 1984)

\begin{abstract}
Mutations in Bacillus subtilis 168 have been isolated that confer resistance to arginine hydroxamate in the presence, but not absence, of ornithine. Seven such Ahor mutants have been studied in detail. In common with certain classes of Ahr mutant (resistant to arginine hydroxamate in the absence of arginine precursors) described previously, these Ahor mutants showed little or no inducibility of enzymes of arginine catabolism. Mutants that showed no inducibility were unable to utilize arginine or ornithine as sole nitrogen source. The only biosynthetic enzyme to show any consistent differences in activity from the parent was ornithine carbamoyltransferase, whose level was slightly elevated in cells grown in the presence of ornithine or citrulline. PBS1 transduction crosses showed that two of the ahor mutations map at the $a h r A$ locus, while a third (unique in its resistance to arginine hydroxamate in the presence of citrulline) mapped at a hitherto undescribed locus closely linked to metC, designated $a h r D$.
\end{abstract}

\section{INTRODUCTION}

Details of molecular mechanisms for regulation of expression of bacterial genes encoding metabolic enzymes are as yet available only for Escherichia coli and Salmonella typhimurium. In the hope of determining whether unfamiliar control devices might be found in an unrelated group, we have been studying as a model system regulation in the pathways of arginine biosynthesis and catabolism in Bacillus subtilis 168 . In this organism, the three readily assayable biosynthetic enzymes $N$-acetylornithine $\delta$-aminotransferase (AcOAT), ornithine carbamoyltransferase (OCT) and argininosuccinase (AS) are repressed in the presence of arginine; the catabolic enzymes arginase (A) and ornithine $\delta$-aminotransferase (OAT) are induced in the presence of arginine or proline, this induction being antagonized (presumably via some form of nitrogen metabolite repression) more effectively by glutamine than ammonia (Harwood \& Baumberg, 1977; Baumberg \& Harwood, 1979). Harwood \& Baumberg (1977) showed that mutants resistant to the arginine analogue arginine hydroxamate $(\mathrm{AH})$ often showed alterations in expression of these enzymes, usually involving loss or non-inducibility of these two catabolic enzymes but sometimes also simultaneous loss of repressibility of the biosynthetic ones. Mountain \& Baumberg (1980) reported that mutations conferring resistance to AH mapped at three unlinked loci on the $B$. subtilis chromosome, ahr $A, B$ and $C$; mutations resulting only in altered expression of the catabolic enzymes mapped at $a h r A$ and $B$, while the two mutations resulting in simultaneous loss of inducibility of the catabolic enzymes and of repressibility of the biosynthetic ones mapped at $a h r C$.

Abbreviations: AcOAT, $N$-acetylornithine $\delta$-aminotransferase; OCT, ornithine carbamoyltransferase; AS, argininosuccinase; $\mathrm{A}$, arginase; OAT, ornithine $\delta$-aminotransferase; $\mathrm{AH}$, arginine hydroxamate; Ahr/s. resistant/sensitive to arginine hydroxamate; Ahor/s, resistant/sensitive to arginine hydroxamate in the presence of ornithine; $\mathrm{Aut}^{+1-}$, able/unable to utilize arginine as sole nitrogen source; Out ${ }^{+1-}$, able/unable to utilize ornithine as sole nitrogen source; ahr- $x$, mutation conferring an Ahr phenotype and not assigned to a particular locus; ahor- $x$, mutation conferring an Ahor phenotype and not assigned to a particular locus; $a h r X$, a locus of $a h r-x$ or ahor-x mutations. 
We describe here the properties of mutants selected as resistant to $\mathrm{AH}$ in the presence of the arginine precursors ornithine or citrulline, but sensitive to it in their absence. The rationale for this procedure was that the enzyme phenotypes of $\mathrm{AH}$-resistant isolates suggested that selection was effectively for intracellular accumulation of arginine (in these cases through deficiency in its breakdown), this then in some way antagonizing the inhibitory effect of $\mathrm{AH}$. It was hoped that the provision of large intracellular levels of ornithine or arginine might, in hypothetical mutants with elevated levels of enzymes mediating the conversion of these to arginine, produce high endogenous levels of arginine and thereby lead to $\mathrm{AH}$ resistance: such mutants might include ones with the hitherto undiscovered phenotype of elevation of levels of biosynthetic enzymes without change in the catabolic ones.

\section{METHODS}

Bacterial strains and media. The bacterial strains used are listed in Table 1. Methods of maintenance, nutrient and minimal media and supplements were as in Mountain \& Baumberg (1980). AH was used at $100 \mu \mathrm{g} \mathrm{ml}^{-1}$; ornithine and citrulline in the presence of $\mathrm{AH}$ were at $1000 \mu \mathrm{g} \mathrm{ml}^{-1}$; and arginine and ornithine as sole nitrogen source were present at $300 \mu \mathrm{g} \mathrm{ml}^{-1}$.

Growth, harvesting of cells, permeabilization of cells by freezing and thawing, and enzyme and protein assays. These were carried out as described by Mountain \& Baumberg (1980), except that AcOAT was assayed by the method of Albrecht \& Vogel (1964).

PBSI-mediated transduction. This was carried out according to Mountain \& Baumberg (1980).

Table 1. Strains of B. subtilis

\begin{tabular}{lll} 
Strain no. & \multicolumn{1}{c}{ Genotype } & \multicolumn{1}{c}{ Source/Reference } \\
EMG50 & Prototroph & Laboratory collection \\
BC369 & argC4 metD1 hisAl aut-I & Young et al. (1969); Mountain \& Baumberg (1980) \\
UL300 & argC4 metD1 hisAl & A. Mountain; Aut transformant of BC369 \\
AM111 & aut-1 & Mountain \& Baumberg (1980) \\
Ahr2-52 & ahrA52 & Mountain \& Baumberg (1980) \\
QB123 & $\operatorname{trpC2~sacA321~ctrAI}$ & Dedonder et al. $(1977)$ \\
QB934 & $\operatorname{trpC2~aroD120~lys-1~}$ & Dedonder et al. $(1977)$ \\
QB944 & $\operatorname{trpC2~purA16~cysA14}$ & Dedonder et al. $(1977)$ \\
MB21 & metC leuA & P. Piggot
\end{tabular}

RESULTS AND DISCUSSION

Isolation and properties of Ahor mutants

Cells of the prototrophic sub-strain EMG50 of B. subtilis 168, suspended in minimal salts-glucose medium, were plated on minimal salts-glucose agar containing $\mathrm{AH}$ together with either ornithine or citrulline. After $24 \mathrm{~h}$, colonies had developed on both media; after a further $24 \mathrm{~h}$, additional small colonies, clearly distinguishable from those appearing earlier, had appeared on $\mathrm{AH}+$ ornithine but not on $\mathrm{AH}+$ citrulline. 217,69 and 407, respectively, of these isolates were patched on the appropriate selecting medium and replicated to minimal salts-glucose plates containing, with ammonia as nitrogen source: $\mathrm{AH} ; \mathrm{AH}+$ ornithine; $\mathrm{AH}+$ citrulline; and without ammonia : arginine or ornithine as nitrogen source. It was found that all the isolates that developed rapidly on $\mathrm{AH}+$ ornithine or citrulline grew on the other selective medium and also, more importantly, on $\mathrm{AH}$ alone. Also, $36 \%$ of those selected on $\mathrm{AH}+$ citrulline and $65 \%$ of those selected on $\mathrm{AH}+$ ornithine had partially or completely lost the capacity to use arginine and/or ornithine as sole nitrogen source (i.e. were $\mathrm{Aut}^{-}$and/or Out ${ }^{-}$). This latter phenotype was common among the isolates selected on $\mathrm{AH}$ alone described by Harwood \& Baumberg (1977) and Mountain \& Baumberg (1980). On the other hand, only $12 \%$ of the isolates that developed slowly on $\mathrm{AH}+$ ornithine grew on $\mathrm{AH}$ alone - these, but not the rest, also grew on $\mathrm{AH}+$ citrulline - though about $75 \%$ showed some deficiency in arginine or ornithine utilization.

These results suggested that the rapidly developing isolates were unlikely to differ significantly from the previously characterized Ahr mutants. The isolates that developed slowly 
Table 2. Growth of Ahor mutants on $A H$ with and without ornithine or citrulline and on arginine or related amino acids as sole nitrogen source

Growth was tested on minimal salts-glucose agar supplemented as shown. Plates were scored after $24 \mathrm{~h}$ at $37^{\circ} \mathrm{C}$.

\begin{tabular}{|c|c|c|c|c|}
\hline & \multicolumn{4}{|c|}{ Growth* on: } \\
\hline & $\mathrm{AH}$ & $\mathrm{AH}+$ ornithine & $\mathrm{AH}+$ citrulline & $\begin{array}{l}\text { Arginine, ornithine or } \\
\text { citrulline as sole } \mathrm{N} \text { source }\end{array}$ \\
\hline EMG50 & - & - & - & $+t$ \\
\hline Ahr 2-52 & ++ & ++ & ++ & - \\
\hline AM111 & - & + & - & - \\
\hline Ahorl & - & + & - & $+\dagger$ \\
\hline Ahor 7 & - & ++ & ++ & $t+\ddagger$ \\
\hline Ahor 10 & - & ++ & - & + \\
\hline Ahor 13 & - & + & - & + \pm \\
\hline Ahor 17, 23, 24 & - & + & - & - \\
\hline
\end{tabular}

on $\mathrm{AH}+$ ornithine and could not grow on $\mathrm{AH}$ alone represented a new phenotype (termed Ahor), and further work was restricted to them. The fact that most of them were to some extent Aut $^{-}$and/or Out ${ }^{-}$indicated that these at least, contrary to our hopes, would probably turn out to be affected in expression of catabolic enzymes. It may also be noted that although the Ahor isolates grew from single cells to colonies in their original selection only in $2 \mathrm{~d}$, on subsequent testing they grew on the same medium in $1 \mathrm{~d}$; this would be consistent with the occurrence of secondary mutations in at least some cases. Seven non-sibling isolates, designated Ahorl, 7, 10, $13,17,23$ and 24 , were chosen for more detailed investigation.

\section{Growth properties of Ahor mutants}

Plate tests were carried out to determine the growth characteristics of the selected Ahor strains on media containing $\mathrm{AH}$ alone or combined in various concentrations with ornithine or citrulline, or including various nitrogen sources in place of ammonia. These tests were carried out simultaneously on (1) the parental EMG50, (2) the Ahr strain Ahr2-52, an example of Class 1 of Harwood \& Baumberg (1977) whose loss of expression of the catabolic enzymes is most extreme, and (3) AM111, a prototrophic derivative of strain BC369, which had been found earlier to be phenotypically Ahor and also Aut ${ }^{-} / \mathrm{Out}^{-}$(Mountain \& Baumberg, 1980). The results are given in Table 2, and can be summed up as follows. AM111 and the new Ahor isolates, with the exception of Ahor 7, are unable to grow on $\mathrm{AH}$, as already indicated, or $\mathrm{AH}+$ citrulline (their capacity to grow on $\mathrm{AH}+$ ornithine is also diminished as the $\mathrm{AH}$ concentration rises or the ornithine concentration drops; data not shown). Ahor7, in sharp contrast, grows well on $\mathrm{AH}+$ citrulline and on the various combinations of $\mathrm{AH}$ and ornithine concentrations tested. As regards use of arginine, ornithine, or citrulline as nitrogen source, there is a gradation among the Ahor isolates from AM111 and Ahor mutants 17, 24 and 24, which are totally unable to utilize any of these, through Ahor 10 to Ahorl and finally Ahor7 and Ahor13. As expected, all strains tested grow well with proline as sole nitrogen source (data not shown).

\section{Enzyme activities in Ahor isolates}

Assays for A, OAT, AcOAT, OCT, AS and protein were performed on permeabilized suspensions of EMG50, AM111 and the seven new Ahor isolates. Specific activities are shown in Table 3 (those for Ahor 17 and 23 are omitted since these isolates behaved like Ahor 24). It is seen that uninduced levels of A (i.e. in cells grown in unsupplemented medium) in the Ahor isolates are either similar to those in EMG50 or less than a factor of two lower; in the presence of arginine, Ahor 1, 10, and 24 show no induction of this enzyme while Ahor 7 and 13 show a 


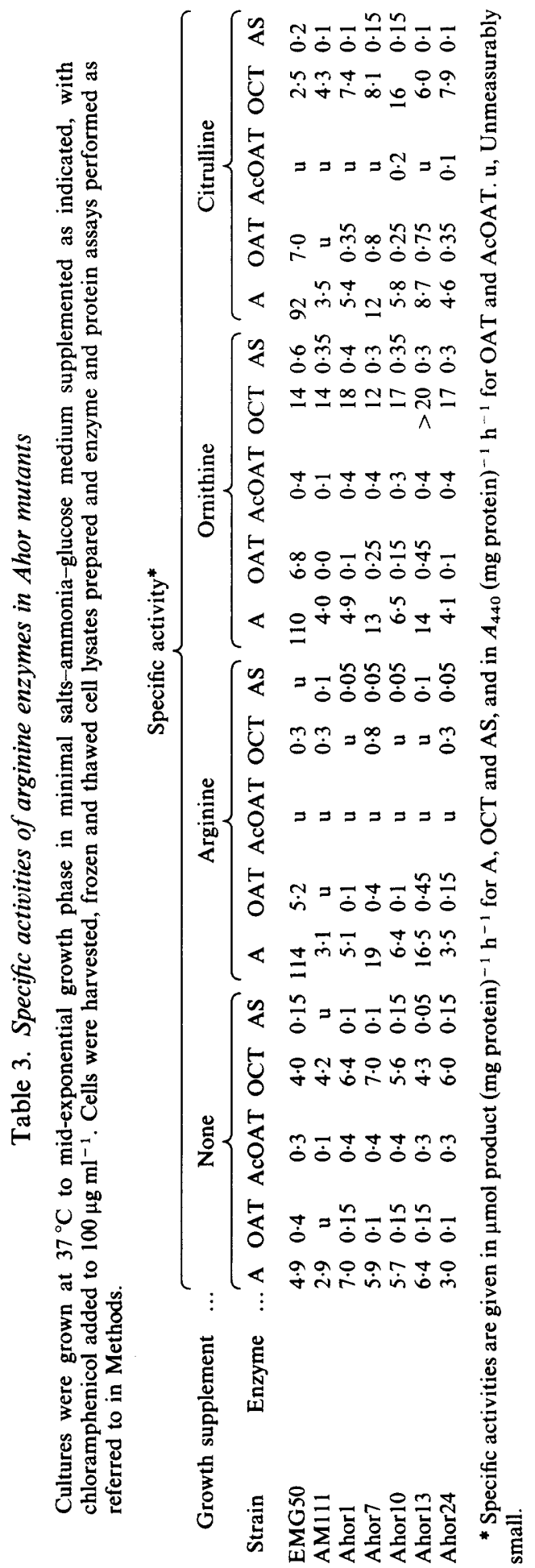


roughly threefold induction, with ornithine and citrulline giving similar results except that the induction is slightly less. OAT behaves similarly to A, though induction by citrulline in all the isolates is two to three times that by arginine and ornithine. These results accord with the superior ability of Ahor 7 and 13 in utilizing these amino acids as sole nitrogen source. These figures are similar to those for Ahr mutant classes 3 and 6 in which there is no, or barely, detectable induction of these enzymes (Mountain \& Baumberg, 1980).

As regards the biosynthetic enzymes, the only clear-cut difference from EMG50 is the elevated level of OCT in citrulline-grown cells of all Ahor strains by comparison with EMG50, by a factor of about two to over six. Possibly significant are the increased levels of OCT (again in comparison to EMG50) in most of the isolates in unsupplemented medium (by, however, a factor of less than two) and in Ahor7 and 17 in medium supplemented with arginine. These OCT levels are reminiscent of those in the sole representative of class 5, Ahrl-120 (Mountain \& Baumberg, 1980), though this strain is less affected in expression of the catabolic enzymes than any described here, induction being about half that found with EMG50. It is clear, in any case, that the enzyme activity characteristics of the Ahor isolates differ at least slightly from those of any of the Ahr isolates previously described.

The AH resistance phenotype of these isolates is therefore roughly in accord with the generalization that this resistance is greater the lower the levels of the catabolic enzymes under inducing conditions. Comparing the data with those of Mountain \& Baumberg (1980), we see that apart from their Class 5 mutant Ahr1-120 (which in their hands seemed to have reverted to AH sensitivity) the only exception to this generalization is the Class 4 mutant Ahr2-48, in which inducibility of A and OAT is under all conditions superior to that of the Ahor strains, and yet which is resistant to AH in the absence of ornithine. Within the Ahor isolates, there is good correlation between inducible levels of the catabolic enzymes and ability to utilize arginine or its precursors as nitrogen source: the isolates with the highest residual inducibility, Ahor 7 and 13, can do so, the others cannot. However, if we include the Ahr strains in the comparison, matters become more complex in that the levels of A and OAT in Ahor 17,23 and 24, which do not utilize arginine or its precursors, are similar to those in members of Class 3 of Mountain \& Baumberg (such as Ahr2-34) which do. Finally, the enzyme activities give no clue to the unique phenotype of Ahor7 in being able to grow on $\mathrm{AH}+$ citrulline.

\section{Mapping of ahor mutations}

To locate the ahor mutations responsible for the Ahor phenotype of these isolates, linkage was first sought in PBS1-mediated transduction with markers found by Mountain \& Baumberg (1980) to be linked to $a h r A, B$ and $C$. Accordingly, PBS1 lysates made on each Ahor strain were used to tranduce recipients QB123 (sacA linked to $a h r A$ and $a h r B$, $\operatorname{ctr} A$ to $a h r B$ ); QB934 (aroD and $l y s A$ both linked to $a h r C$ ); QB944 (cys $A$ and purA both linked to $a h r A$ ); and UL300, this being included since the only biosynthetic enzyme affected in the Ahor set is the OCT encoded by $\arg C$, this gene being linked to met $D$.

No linkage was found of any ahor marker with sacA, $\operatorname{ctrA}$, aroD or lysA. Also, mutations ahor$1,-10,-13$ and -24 , showed no linkage with any marker tested. It should be noted, however, that in several cases particular combinations of lysate and recipient gave very few transductants so that weak linkage might have been missed. Results in cases where linkage was detected are given in Table 4. It is seen that ahor-17 and -23 show the linkage with cys $A$ and pur $A$ characteristic of $a h r A$ mutations; when $\mathrm{Pur}^{+}$, but not $\mathrm{Cys}^{+}$, transductants were scored separately for the Ahor/s and Out ${ }^{+1-}$ properties, combinations of these different from either parent were observed yielding the complicated picture described by Mountain \& Baumberg (1980). ahor-7 was found to be cotransducible with metD1 and $\operatorname{argC4}$, the distribution of unselected markers indicating the order metD-argC-ahor-7 which is the only one yielding Ahos $\mathrm{Met}^{-}$and $\mathrm{Ahos} \mathrm{Arg}^{-}$as the rarest recombinant classes among transductants selected as $\mathrm{Arg}^{+}$and $\mathrm{Met}^{+}$, respectively. $a h r-7$ therefore appeared to map at a new $a h r$ locus which we name $a h r D$, on the side of $\arg C$ opposite to met $D$. A suitable marker in this region with which to test linkage is metC. Accordingly, PBS1 grown on Ahor7 was used to transduce MB21 to $\mathrm{Met}^{+}$. As indicated in Table 4, strong linkage (about $95 \%$ ) was found. We have not attempted to map 
Table 4. Results of transductional crosses with Ahor donors

\begin{tabular}{lccccc}
\multirow{2}{*}{ Recipient } & $\begin{array}{c}\text { Selection } \\
\text { for: }\end{array}$ & Donor & $\ldots$ & \multicolumn{3}{c}{ Ahor7 } & Ahor17 & Ahor23 \\
QB944 & CysA $^{+}$ & & $0 / 34$ & $8 / 52$ & $2 / 25$ \\
& PurA $^{+}$ & & $0 / 46$ & $46 / 55$ & $29 / 37$ \\
UL300† & ArgC $^{+}$ & $33 / 119 \ddagger$ & $0 / 111$ & $0 / 111$ \\
& MetD $^{+}$ & $11 / 148 \ddagger$ & NT & NT \\
MB21 & MetC $^{+}$ & $68 / 71$ & NT & NT
\end{tabular}

* NT, Not tested.

$\dagger$ The Ahor/s character was scored in transductants of this recipient with $\mathrm{AH}$ at $75 \mu \mathrm{g} \mathrm{ml}^{-1}$.

$\ddagger$ The frequencies of the various unselected marker combinations in these transductants were as follows. Selection for $\mathrm{ArgC}^{+}$: A hor MetD ${ }^{+} 20$; Ahor MetD ${ }^{-}$13; Ahos MetD ${ }^{+}$84; Ahos MetD- 2. Selection for MetD ${ }^{+}$: $\mathrm{Ahor}_{\mathrm{ArgC}}^{+} 11 ; \mathrm{Ahor}^{\mathrm{ArgC}^{-}}$0; Ahos $\mathrm{ArgC}^{+} 118$; Ahos $\mathrm{ArgC}^{-} 19$.

ahrD7 any further; however, it is tempting to speculate whether it might show any connection with the $\operatorname{pro}(A B)$ locus reported by Harwood (1974) and Buxton (1980) to be strongly linked to $m e t C$. The phenotype of $\operatorname{pro}(A B)$ mutants, a requirement for arginine or proline, indicates a block in the proline biosynthetic pathway between glutamate and glutamic- $\gamma$-semialdehyde; this pathway operates in the reverse direction in the catabolism of arginine or proline to glutamate. This locus also appears to be the site of mutations conferring resistance to the proline analogue $\mathrm{L}$ azetidine-2-carboxylate (Gallori et al., 1978), which may be regulatory in nature.

We thank R. Dedonder, J.-A. Lepesant and P. Piggot for strains. J. McChesney assisted in some of the mapping. This work was supported by MRC grant G974/223/B to S. B.

\section{REFERENCES}

Albrecht, A. M. \& Vogel, H. J. (1964). Acetylornithine $\delta$-transaminase: partial purification and repression behaviour. Journal of Biological Chemistry 239, $1872-1876$.

Baumberg, S. \& Harwood, C. R. (1979). Carbon and nitrogen repression of arginine catabolic enzymes in Bacillus subtilis. Journal of Bacteriology 137, 189-196.

Buxton, R. S. (1980). Selection of Bacillus subtilis 168 mutants with deletions of the PBSX prophage. Journal of General Virology 46, 427-437.

Dedonder, R. A., LePesant, J.-A., LePeSANT-KeJZlarova, J., Billault, A., Steinmetz, M. \& Kunst, F. (1977). Construction of a kit of reference strains for rapid genetic mapping in Bacillus subtilis 168 . Applied and Environmental Microbiology 33, 989-993.

Gallori, E., Bazzicalupo, M., Parisi, B., Pedaggi, G. \& Polsinelli, M. (1978). Resistance to (L)azetidin-2-carboxylic acid in Bacillus subtilis. Bio- chemical and Biophysical Research Communications 85, $1518-1525$.

HARWOOD, C. R. (1974). Genetic control of arginine enzymes in the bacterium Bacillus subtilis. Ph.D. thesis, University of Leeds, UK.

Harwood, C. R. \& Baumberg, S. (1977). Arginine hydroxamate-resistant mutants of Bacillus subtilis with altered control of arginine metabolism. Journal of General Microbiology 100, 177-188.

Mountain, A. \& Baumberg, S. (1980). Map locations of some mutations conferring resistance to arginine hydroxamate in Bacillus subtilis 168. Molecular and General Genetics 178, 691-701.

Young, F. E., Smith, C. \& Reilly, B. E. (1969). Chromosomal location of genes regulating resistance to bacteriophage in Bacillus subtilis. Journal of Bacteriology 98, 1087-1097. 